\title{
Design of the Pedometer Based on MATLAB and Android Smartphone Sensor
}

\author{
Na Yang ${ }^{1, a^{*}}$ and Yanan $\mathrm{Hu}^{1, b}$ \\ ${ }^{1}$ Mechanical and electrical technology department,Xijing University, Shaanxi Xi'an ,China \\ a515569778@qq.com \\ * the corresponding author
}

Keyword: Smartphones; A pedometer; The wireless network; The filter

\begin{abstract}
This paper designs a pedometer based on MATLAB and the Android smartphones sensor. First analysis the change law of acceleration of the human body when people exercise, obtaining 3D acceleration through mobile phone sensor, and then send to the computer which running MATLAB software in the form of UDP packets through the wireless network by the sensorudp APP, and use the powerful MATLAB software to decode the packets to get the acceleration, and then execute the filter and spline interpolation, and get the number of steps, the distance and energy consumption, which can provide the basis to judge the movement.
\end{abstract}

\section{Introduction}

Since the launch of the Android smartphone, its built-in sensors have gradually increased and the functions realized by the sensors are increasingly diverse. Android smartphones have greatly satisfied users' demand for smartphone functionality. From the kinds of games rely on gravity sensor, to rely on distance sensor implementation calls out the screen, and then to the compass under the function of electronic compass, and so on, a small Android smartphone based on various sensors to realize the function of a number of interesting. So the phone is no longer a simple communication tool, but a portable electronic device with integrated functions. In this case, the application of various sensors on the phone comes into being. The G-sensor in this paper is to use Android smartphone sensors, combined with the powerful calculation function of MATLAB software, we design a pedometer, movement is obtained by analysis of sensor data processing steps, further calculation can be data, such as speed, distance and energy consumption. In the context of general public health awareness, various kinds of sports equipment become hot, and the application of the pedometer can help improve the movement effect and achieve the goal of healthy movement.

\section{The Principle of Pedometer}

The first step is to understand the posture of walking. Based on the study characteristics of pace, considering the human walking feet, legs, waist and arms are in motion, their movement produces the corresponding acceleration, the trend is constantly ups and downs of approximate sine process. The frequency of the sinusoidal wave can be measured by the number of steps in motion, which can then calculate the speed, distance, and consumption of calories. The acceleration from the foot is the most accurate, but considering the convenience of carrying, choosing to use the waist movement to detect the number of steps. Because of the vertical movement of the waist up and down in the middle of the walk, a higher and lower fluctuation of the acceleration is produced. Because of the acceleration sensor in the smartphone(refer with:Fig. 1). It has three axes that correspond to the three directions of human motion, so the first rule is that the human body is moving in the $\mathrm{Y}$-axis, the right and the left motion is the $\mathrm{X}$-axis, and the upper and lower motion is the z-axis. No matter how the cell phone is placed, the acceleration value and the sum of the three directions will be detected and the acceleration a: 


$$
|a|=\sqrt{a_{x}^{2}+a_{y}^{2}+a_{z}^{2}}
$$

When the phone is still stationary, the ideal value of a is the gravitational acceleration $\mathrm{g}$. When an action is detected, the a value changes in the $\mathrm{g}$ value, and in order to calculate the convenience, $\mathrm{a}=\mathrm{a}-\mathrm{g}$ can be taken, and the a value fluctuates from 0 to 0 . With the focus of the body as a reference point, only the $\mathrm{z}$ axis is considered, and the walking process can be divided into two stages:The center of gravity is from the lowest to the highest and the center of gravity is from the highest to the lowest. In the process of center of gravity from the lowest to the highest, the body of the $\mathrm{z}$ axis speed from zero to a certain value, and then from the value is reduced to zero, the whole process speed is positive, the direction of $\mathrm{z}$ axis is the direction. So, the change in acceleration should go from $a_{z}=-g\left(\mathrm{~m} / \mathrm{s}^{2}\right)$ to $a_{z}=-\left(g+\Delta_{a}\right)\left(m / s^{2}\right)$ and then back to $a_{z}=-g\left(m / s^{2}\right)$, which $\Delta_{a}$ is positive. In the process of center of gravity from the highest to the lowest, the human body the $\mathrm{z}$ axis speed from zero to a certain value, and then from the value is reduced to zero, the whole process speed is negative, namely the direction for the $\mathrm{z}$ axis direction. So, the change in acceleration should go from $a_{z}=-g\left(\mathrm{~m} / \mathrm{s}^{2}\right)$ to $a_{z}=-g\left(\mathrm{~m} / \mathrm{s}^{2}\right)$ and go back to $a_{z}=-g\left(\mathrm{~m} / \mathrm{s}^{2}\right)$, where $\Delta_{a}$ is positive. From this, we can conclude that the change process in A one cycle is as close as shown in Fig. 2:

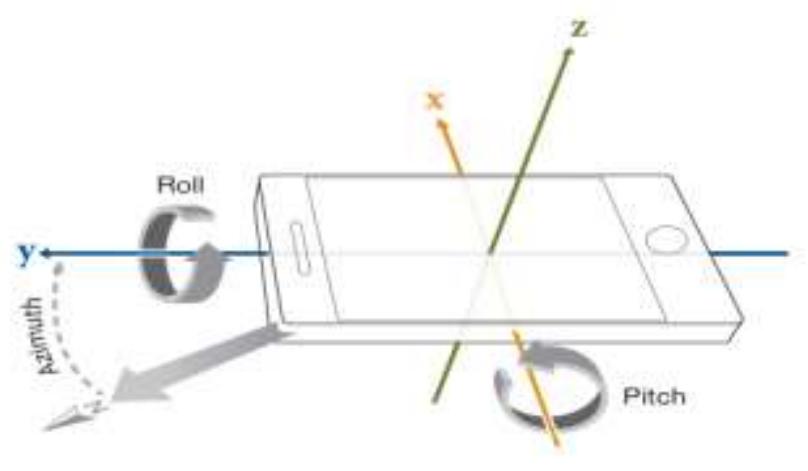

Figure 1. 3D acceleration direction of mobile phone

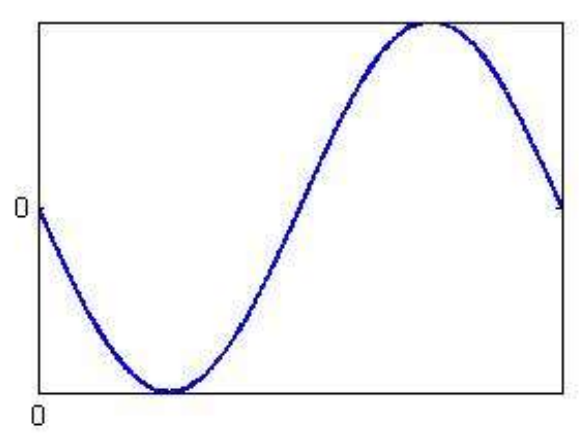

Figure 2. change law of $\mathrm{A}$

It can be seen that the change curve of parameter A is approximately A sinusoidal signal, so only the frequency of the sinusoidal signal is required to know the total number of steps taken.

\section{To Establish Communication}

MATLAB support from Android devices to obtain data of the built-in sensors, through MATLAB support for Android sensor package, you can record data from sensor supported by Android devices or see the latest available data, and then through the analysis of data to develop different applications. The premise of this is that the sensorudp APP must be installed on the phone in advance, and the computer that runs the MATLAB software and the smart phone that installs the sensorudp APP are in the same network. The Sensorudp mentioned in this article is a smartphone application developed by Takashi SASAKI (shown in Fig. 3), which can obtain the data of various sensors of the phone, including the acceleration of three-dimensional direction. Angular velocity in $3 \mathrm{~d}$ direction; Azimuth Angle, heel Angle and pitch Angle; Dimensions, longitude, height and speed; 


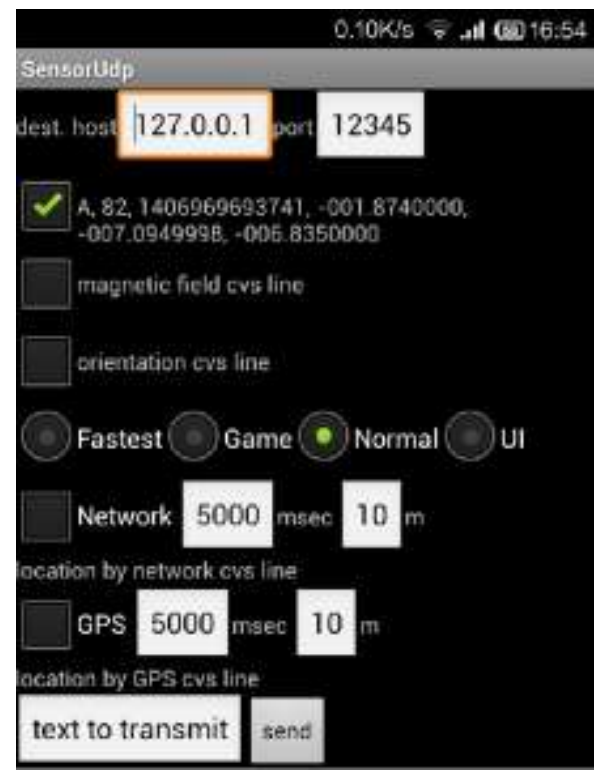

Figure 3. Interface of sensorudp APP

\section{Data Measurement and Analysis}

The measurement and analysis of data is a key step in the design of the pedometer. Through the whole process, the specific pedometer procedure is shown in Fig. 4.

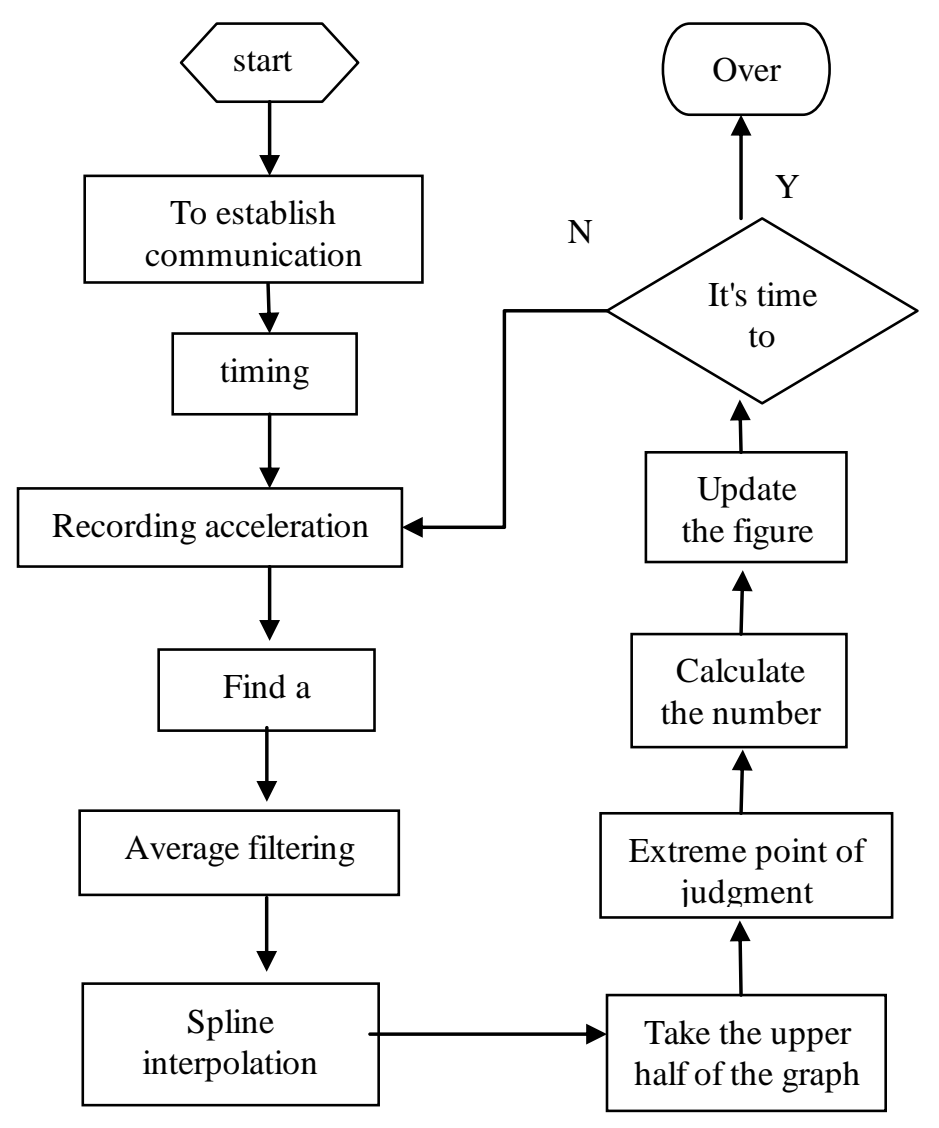

Figure. 4 Program flow chart

After the communication is established, the sensor data can be obtained, and the local gravity acceleration must be obtained first. Because the gravitational acceleration in one place is associated with latitude and altitude, the acceleration of gravity varies from place to place. At the same time, the 
influence of the phone itself and the surrounding environment can interfere with the detection of the sensor. In this paper, the experimental method is used to obtain the detection value and the average value of the g-sensor sensor in the smartphone. Specific method is the phone still on the ground, take the position of the three different at each location will phone positive, negative, place and the measurement, the side of data receiving 20 seconds at a time, a total of measuring nine times, each time the measurement will be a series of values, the normalized, and will finally get nine mean to calculate the sum of the mean, the value can be viewed as the local acceleration of gravity.

\section{Conclusion}

In this paper, based on the analysis of the change of acceleration of human motion, the MATLAB software and Android smartphone sensor are combined to establish a wireless network between the two, and realize the communication of data. The acceleration signals obtained from the sensor are filtered and interpolated, and the number of moving steps is consistent with the number of steps recorded. With the rise of smart phones, all kinds of mobile sensors is becoming more and more powerful, if combined with the powerful functions of MATLAB software, will provide convenience for the development of various applications, this article is based on a beneficial attempt.

\section{References}

[1] Liu Huazhou. Research on image matching technology based on smart phone sensor[D]. Xi 'an university of electronic science and technology 2013.

[2] Zhang Ye. The implementation and application of WIFI based on Android platform[D]. Inner Mongolia university 2013.

[3] Zhang Ning. Implementation of mobile terminal data acquisition based on Android[D]. Inner Mongolia university 2013.

[4] Li Xiaoyang. WiFi technology and its application and development[J]. Information technology 2012(02)

[5] Tang Jianhua,Chen Jinying. The application of WiFi transmission technology in control field[J]. Information and communication. 2013(04) 